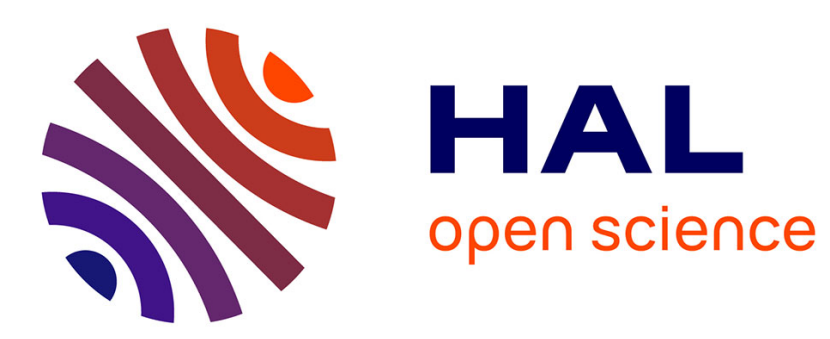

\title{
Condition-Based Predictive Maintenance in the Frame of Industry 4.0
}

Alexandros Bousdekis, Gregoris Mentzas

\section{To cite this version:}

Alexandros Bousdekis, Gregoris Mentzas. Condition-Based Predictive Maintenance in the Frame of Industry 4.0. IFIP International Conference on Advances in Production Management Systems (APMS), Sep 2017, Hamburg, Germany. pp.399-406, 10.1007/978-3-319-66923-6_47 . hal-01666184

\section{HAL Id: hal-01666184 \\ https://hal.inria.fr/hal-01666184}

Submitted on 18 Dec 2017

HAL is a multi-disciplinary open access archive for the deposit and dissemination of scientific research documents, whether they are published or not. The documents may come from teaching and research institutions in France or abroad, or from public or private research centers.
L'archive ouverte pluridisciplinaire HAL, est destinée au dépôt et à la diffusion de documents scientifiques de niveau recherche, publiés ou non, émanant des établissements d'enseignement et de recherche français ou étrangers, des laboratoires publics ou privés. 


\title{
Condition-based Predictive Maintenance in the Frame of Industry 4.0
}

\author{
Alexandros Bousdekis ${ }^{1}$ and Gregoris Mentzas ${ }^{1}$ \\ ${ }^{1}$ Information Management Unit (IMU), Institute of Communication and \\ Computer Systems (ICCS), National Technical University of Athens (NTUA), Greece \\ \{albous, gmentzas\}@mail.ntua.gr
}

\begin{abstract}
The emergence of Industry 4.0 leads to the optimization of all the industrial operations management. Maintenance is a key operation function, since it contributes significantly to the business performance. However, the definition and conceptualization of Condition-based Predictive Maintenance (CPM) in the frame of Industry 4.0 is not clear yet. In the current paper, we: (i) explicitly define CPM in the frame of Industry 4.0 (alternatively referred as Proactive Maintenance); (ii) develop a unified approach for its implementation; and, (iii) provide a conceptual architecture for associated information systems.
\end{abstract}

Keywords: Proactive Maintenance; E-maintenance; Event processing.

\section{Introduction}

Industry 4.0 indicates the flexibility that exists in value-creating networks which enables machines and plants to adapt their behavior to changing orders and operating conditions through self-optimization and reconfiguration. To do this, they perceive information, derive findings and change their behavior accordingly. At the same time, they store knowledge gained from experience with the aim to implement distributed and interconnected production facilities in future smart factories [1]. Industry 4.0 combines computer science with electrical and mechanical engineering and is considered to be a revolution on a business level and an evolution on a technological level.

Maintenance is a key operation function, since it is related to all the manufacturing processes and focuses not only on avoiding the equipment breakdown but also on improving business performance in terms of productivity, quality and logistics management [2,3]. Existing maintenance strategies can broadly be distinguished to three categories: (a) Breakdown Maintenance; (b) Time-based Preventive Maintenance; (c) Condition-based Predictive Maintenance (CPM) [2]. Since products have become more and more complex due to the evolution of technology, the costs of time-based preventive maintenance have increased and CPM has evolved as a novel lever for maintenance management [4]. However, there is not a clear classification in literature, while CPM in the context of Industry 4.0 has not been concretely defined. Currently, even large manufacturing companies have not developed a complete CPM strategy and appropriate sensor-driven, real-time systems in order to utilize its benefits [2]. 
Existing CPM solutions suffer from several limitations: (i) Most of them focus on product maintenance, i.e. on the service stage of the Product Lifecycle Management (PLM) (e.g. warranty failures) and not on industrial maintenance, i.e. on the manufacturing stage of the PLM; (ii) They are mainly based upon physical, domain-specific models that are not easily extensible for other equipment or for other industries; (iii) They rarely exploit big data processing infrastructures for real-time, sensor data, since they usually use batches of data, while the level of data analytics maturity is usually low; (iv) Each one of them focuses on a specific aspect of CPM (e.g. condition monitoring, diagnostics, etc.) instead of having a unified approach for covering all the phases and industrial operations-related aspects.

The objectives of the current paper are: (i) to explicitly define CPM in the frame of Industry 4.0; (ii) to develop a unified approach for its implementation; and, (iii) to provide a generic reference conceptual architecture for the development of associated information systems. The rest of the paper is organized as follows. Section 2 discusses the literature review. Section 3 includes the definition of CPM in the frame of Industry 4.0, i.e. Proactive Maintenance. Section 4 outlines a unified approach for Proactive Maintenance implementation, while Section 5 presents a generic reference conceptual architecture. Section 6 concludes the paper and discusses the future work.

\section{$2 \quad$ Literature Review}

\subsection{Condition-based Predictive Maintenance and E-maintenance}

$\mathrm{CPM}$ is an evolving maintenance strategy that is increasingly gathering the interest of modern manufacturing companies by utilizing the capabilities of condition monitoring. Condition monitoring is usually realized with equipment-installed sensors, which have the capability of measuring with high frequency a multitude of parameters [5] leading to processing and storage of a huge amount of data. Big data pose challenges to the subsequent processing pipeline of data analysis, knowledge extraction and decision making [2]. The classical industrial view of CPM is mainly focused on the use of condition monitoring techniques such as vibration analysis, thermography, acoustic emission or tribology [5]. The recent developments of maintenance management lead to a physical understanding of a system's useful life and health state prediction through dynamic pattern recognition in various available data sources. Despite the plethora of CPM frameworks existing both in the academic and the industrial realms, there is still a large gap for effective implementation of CPM extensively in industry, mainly due to complexity of these solutions and their life cycle [4].

E-maintenance refers to the convergence of emerging information and communication technologies with systems which take into account the resources, services and management to enable decision making in a proactive way [3] and thus, to enhance and extend the CPM framework [4]. E-maintenance incorporates emerging technologies capable of optimizing maintenance-related workflows and integrating business performance with other components of e-enterprise [4]. The development of emaintenance systems can be distinguished in two chronological periods which have 
different characteristics. The first wave of appearance, development and deployment of e-maintenance concepts and prototypes was during the period 2003-2008 (see e.g. [6-8]). The second wave of e-maintenance paradigms has appeared in 2014 due to the emerging opportunities of the Industrial Internet of Things (IIoT), big data infrastructures and communication devices, but also due to the increasing financial pressures leading to a significant demand of eliminating maintenance costs (see e.g. [9-10]).

\subsection{Industrial Internet of Things, Big Data and Proactive Event Processing}

A CPM strategy implementation requires a complete methodology as well as appropriate information systems capable of processing information captured by sensors in order to provide added value insights [2]. It can take advantage of IIoT advances for handling failure uncertainty, since the stochastic nature of the equipment degradation process leads to high uncertainty in decision making [11]. Since the manufacturing domain is driven by events gathered through sensors, there is the need for event monitoring and big data processing information systems [12]. Modern manufacturing companies have started to collect and store operations-related data or even utilize technological infrastructures and information systems for monitoring various parameters that are known to affect equipment condition and detecting early warning signals that machines or systems are degrading or in danger of breakdown [2]. However, the strategic value of data analysis in the modern dynamic manufacturing enterprises should be increased by augmenting the level of data analytics maturity, through efficiently processing of real-time big data and supporting multi-directional knowledge flows, in order to enable the maintenance strategy transformation.

Proactivity in terms of information systems is driven by predictions, leading to increased situation awareness and decision making capabilities ahead of time. Proactive computing extends the reactive pattern known as 'Sense-and-Respond' [13] or 'Detect-and-Act' [14] to a novel pattern which consists of four phases [15]: Detect, Predict, Decide, Act. This proactive principle can be the base for a unified CPM framework and for its implementation with an appropriate information system by mapping the maintenance and other industrial operations (e.g. warehouse management, production planning, quality improvement) to these phases.

\section{Definition of Proactive Maintenance}

CPM in the frame of Industry 4.0 should be clearly defined taking into account the state-of-the-art theories, concepts and technologies in order to pave the way for the complete transformation of manufacturing enterprises from reactive to proactive. We argue that Proactive Maintenance indicates the CPM in the frame of Industry 4.0 in the sense that it brings together maintenance management and proactivity in terms of information systems. Proactive Maintenance has a managerial and a technological perspective. From a managerial point of view, its implementation requires the identification of the need for a different maintenance strategy through feasibility studies [16] as well as the radical change of maintenance-related business processes and op- 
erations in all the enterprise organizational levels (operational, management, strategic). From a technological point of view, it requires appropriate technologies and information systems for effectively supporting the Industry 4.0 principles. Therefore, Proactive Maintenance should include the following characteristics:

- IoT-based Condition Monitoring. Condition monitoring is applied with sensors at a component, machine or production process level. The decisions about their type and their distribution (placement) are affected by the manufacturing system examined. These hardware and/or software sensors generate huge amounts of realtime data (big data) in the context of IIoT which are further processed through appropriate infrastructures. The term "predictive maintenance" that is often used does not necessarily include real-time condition monitoring through sensors.

- Event-driven Architecture. Event processing is used to process massive primitive events and get valuable high level information from them by continuously monitoring the event flow. Therefore, through the event triggers, event-driven infrastructures are able to handle big data in a scalable and efficient way.

- Prognosis Lifecycle. Prognostic lifecycle covers all the maintenance phases, through which information is processed; from signal processing and diagnostics till prognostics and maintenance decision making along with continuous improvement during actions implementation. Predictions about the future equipment condition, on the basis of which mitigating actions can be applied ahead of time, constitute the backbone of CPM. They can be realized with associated predictive event processing agents. The term "Condition Based Maintenance" that is usually used does not necessarily include predictions, since it may refer to (near) real-time diagnostic outcomes, i.e. detection of the current condition.

- Proactive Computing. Proactive event processing makes it possible to anticipate potential issues during process execution and thereby enables proactive process management, i.e. to decide and act on the basis of real-time predictions. The proactive event-driven applications are subjected to the proactive principle. A proactive situation includes a future event, a predictive pattern, the probability distribution function of the event occurrence, a list of mitigating actions and costs (e.g. the cost of the future event, the costs of actions as function of implementation time) [15].

- E-maintenance Support. The e-maintenance concept is linked to the CPM framework, since it provides the communication and technological background for real-time data processing and information exposure to the users and thus, it can support all the phases of the proactive principle. E-maintenance applications and platforms can facilitate proactivity and further advance to a greater value with the development of Cyber-Physical Systems, while they are able to utilize an eventdriven architecture for scalable sensor-generated big data processing.

- Interaction with other Industrial Operations. Since, every change in industrial operations affects the others, CPM should be considered along with its interactions with the other operations. A reduction in production, quality and inventory costs is considered as one of the most important indirect benefits of CPM [11]. For instance, due to the available real-time prognostic information, predictive maintenance actions along with quality improvement and production activities can be recommended and spare parts can be ordered just in time. 


\section{A Unified Approach for Proactive Maintenance}

The proposed unified approach combines and extends existing CPM approaches [2]. It aims to frame and conceptualize Proactive Maintenance in order to enable manufacturing companies to fully exploit the availability of huge amounts of realtime and historical data with respect to the implementation of Proactive Maintenance by advancing their data analytics maturity. Unification can be achieved by bringing together approaches, tools and services each one of which implements a different phase of the Proactive Maintenance framework in order to effectively support different enterprise management layers, i.e. operational, management, strategic. Moreover, it enables data aggregation and interpretation as well as information sharing throughout the whole organization, both horizontally and vertically.

The systematic representation of any Proactive Maintenance solution allows its application in the production process of any manufacturing company regardless their processes, products and physical model used, while additional aspects (e.g. company policies) can be addressed with context-awareness. The proposed framework aims to cover the whole prognostic lifecycle along with their interactions with quality management, production planning and logistics decisions. The overall unified approach for Proactive Maintenance is depicted graphically in Fig. 1. Each conceptual block should incorporate an e-maintenance service or tool implementing appropriate methods and algorithms. The conceptual blocks are communicated among them through events. The event-driven approach enables the combination of model-based, knowledge-based and data-driven algorithms and methods existing in each conceptual block provided that each one is able to provide the required output that the next one requires. The scope of each conceptual block is explained below:

- Signal Processing: It consists of the "Data Acquisition" and "Data Manipulation" steps, being performed in a modular way utilizing new emerging technologies. It should be able to connect and acquire data from heterogeneous data sources and integrate them for further processing.

- Diagnosis: It consists of the "State Detection" and "Health Assessment" steps. It may include real-time model-based, knowledge-based and/or data-driven methods, embedded in appropriate and continuously processing software in order to recognize the presence of an unusual state with respect to a model of 'normal' behavior.

- Prognosis: It includes state prediction of a whole system or components with respect to a mechanical system, e.g. prediction about the time-to-failure and the probability distribution function of the failure occurrence, the Remaining Useful Life (RUL), the Remaining Life Distribution (RLD). The analysis can be carried out by model-based, knowledge-based and/or data-driven algorithms.

- Maintenance Decision Making: It includes decision making algorithms for taking decisions automatically or for providing recommendations ahead of time, on the basis of real-time prognostic information and maintenance-related expert knowledge. Thus, the optimal mitigating (perfect or imperfect) maintenance actions and the optimal times for their implementation are derived. The decisions can be continuously improved through a Sensor-Enabled Feedback (SEF) mechanism. 
- Maintenance Action Implementation: It includes a real-time feedback mechanism for gathering, storing, aggregating, analyzing and visualizing data and information with respect to the actual actions implementation. The actions are implemented based on the generated decisions either automatically or by humans receiving the recommendations. The sensor-generated data are further processed in order to continuously update and improve the diagnostic, prognostic (through FMECA) and decision (through SEF) models and their continuous learning capability.

- FMECA: Apart from their visualization, in order to further be processed, the actions-related data and information feed into the Failures Modes Effects and Criticality Analysis (FMECA) mechanism, which incorporates algorithms for the identification of potentially relevant and critical failures modes. FMECA can be implemented either on a physical model base or on a data analytics base.

- Industrial Operations Management: It is based upon data and information about production planning, logistics management and quality management. They are stored in company's systems (e.g. ERP, MES) and are gathered in order to consider the industrial operations affected by maintenance processes (e.g. quality levels, production activities, spare parts inventory) and optimize the overall business performance. The data are processed according to the proactive principle and interact with the e-maintenance services. Examples of their utilization could be productionrelated predictions, joint maintenance and inventory optimization algorithms, quality-related constraints in the maintenance utility function optimization, etc.

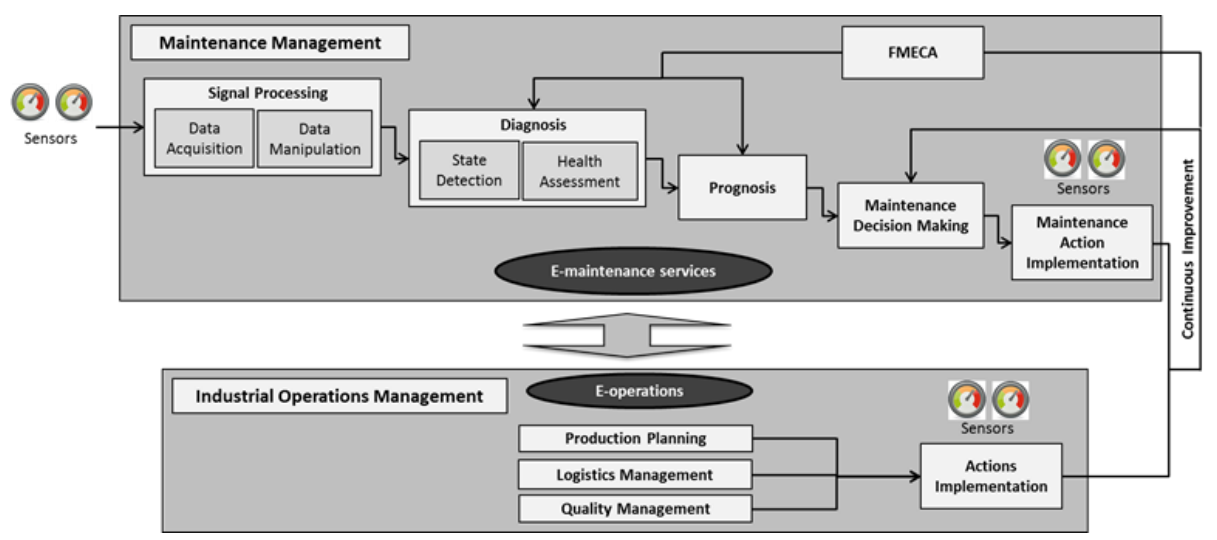

Fig. 1. The Unified Approach for Proactive Maintenance

\section{Conceptual Architecture of a Proactive Maintenance System}

The conceptual architecture of a Proactive Maintenance intelligent information system is shown in Fig. 2. It consists of 3 layers which are further described below:

- User Interaction Layer. The user interaction layer occupies the top level of the architecture and includes a configuration and visualization dashboard that supports the configuration of the architecture and the embodiment of the appropriate expert 
knowledge, as well as the visualization of the current and the predicted equipment behavior, the generated recommendations, etc. with the use of appropriate graphs.

- Real-time Processing Layer. The real-time processing layer deals with the continuous processing of sensor data according to the Proactive Maintenance framework by applying the proactive principle of information systems in both the emaintenance and e-operations services, which can interact between them with the aim to schedule the maintenance activities together with the production, the quality and the logistics ones.

- Data Layer. The data layer of the architecture includes a Database Abstraction Layer and houses a relational database engine where all information needed by the other two layers is stored and retrieved.

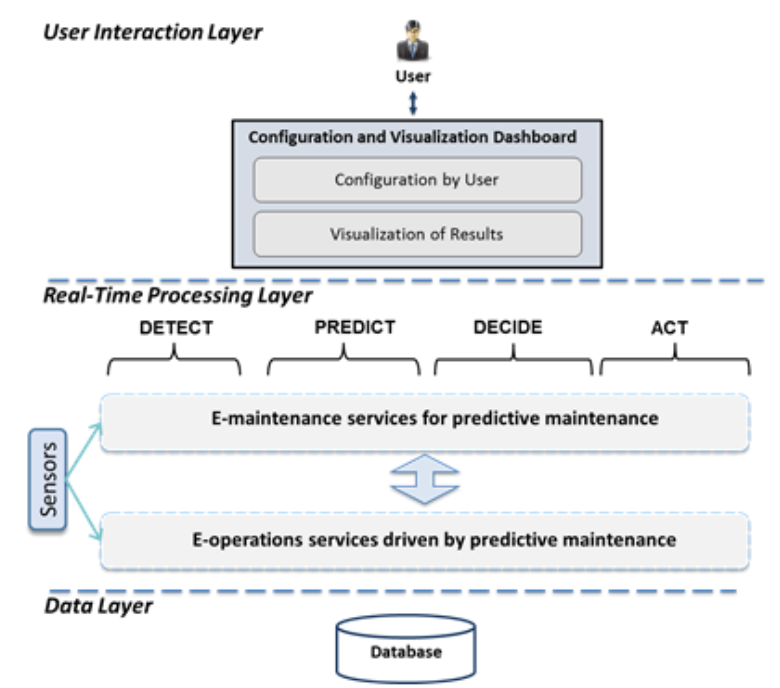

Fig. 2. The Conceptual Architecture of a Proactive Maintenance System

\section{Conclusions and Future Work}

In the current paper, we explicitly defined CPM in the frame of Industry 4.0, which can be referred as Proactive Maintenance, we developed a unified approach for its implementation and we provided a generic reference conceptual architecture for the development of Proactive Maintenance systems with the aim to maximize the expected utility of manufacturing firms and to exploit the full potential of predictive maintenance management, condition monitoring, sensor-generated big data processing, e-maintenance and proactive computing. Regarding our future work, we plan to develop a unified Proactive Maintenance information system by using, extending, developing and integrating new e-maintenance services and tools addressing the various phases of the aforementioned framework for exploiting the full potential of Proactive Maintenance. Then, we aim to validate our approach in industrial environment. 
Acknowledgements. This work is partly funded by the European Commission project FP7 ProaSense "The Proactive Sensing Enterprise" (612329).

\section{References}

1. Lee, J., Bagheri, B., Kao, H. A.: A cyber-physical systems architecture for industry 4.0based manufacturing systems. Manufacturing Letters, 3, 18-23 (2015).

2. Bousdekis, A., Magoutas, B., Apostolou, D., Mentzas, G.: A proactive decision making framework for condition-based maintenance. Industrial Management \& Data Systems, 115(7), 1225-1250 (2015).

3. Fumagalli, L., Macchi, M.: Integrating maintenance within the production process through a flexible E-maintenance platform. IFAC-PapersOnLine, 48(3), 1457-1462 (2015).

4. Guillén, A. J., Crespo, A., Gómez, J. F., Sanz, M. D.: A framework for effective management of condition based maintenance programs in the context of industrial development of E-Maintenance strategies. Computers in Industry, 82, 170-185 (2016).

5. ISO, 2012: ISO 13379-1:2012, Condition monitoring and diagnosis of machines-Data interpretation and diagnosis techniques-Part 1: General guidelines.

6. Djurdjanovic, D., Lee, J., Ni, J.: Watchdog Agent-an infotronics-based prognostics approach for product performance degradation assessment and prediction. Advanced Engineering Informatics, 17(3), 109-125 (2003).

7. Bangemann, T., Rebeuf, X., Reboul, D., Schulze, A., Szymanski, J., Thomesse, J. P., Thron, M., Zerhouni, N.: PROTEUS - Creating distributed maintenance systems through an integration platform. Computers in Industry, 57(6), 539-551 (2006).

8. Levrat, E., Iung, B., Crespo Marquez, A.: E-maintenance: review and conceptual framework. Production Planning \& Control, 19(4), 408-429 (2008).

9. Papa, G., Zurutuza, U., Uribeetxeberria, R.: Cyber physical system based proactive collaborative maintenance. In: Smart Systems and Technologies (SST), International Conference on, pp. 173-178. IEEE (2016).

10. Oelker, S., Lewandowski, M., Alla, A. A., Thoben, K. D.: Preactive Maintenance-A Modernized Approach for Efficient Operation of Offshore Wind Turbines. In: Dynamics in Logistics, pp. 323-331. Springer International Publishing (2016).

11. Van Horenbeek, A., Buré, J., Cattrysse, D., Pintelon, L., Vansteenwegen, P.: Joint maintenance and inventory optimization systems: A review. International Journal of Production Economics, 143(2), 499-508 (2013).

12. Babiceanu, R. F., Seker, R.: Big Data and virtualization for manufacturing cyber-physical systems: A survey of the current status and future outlook. Computers in Industry, 81, 128137 (2016).

13. Alexopoulos, K., Makris, S., Xanthakis, V., Sipsas, K., Chryssolouris, G.: A concept for context-aware computing in manufacturing: the white goods case. International Journal of Computer Integrated Manufacturing, 29(8), 839-849 (2016).

14. Schulte W.R.: How to choose design patterns for event processing applications. Gartner report \#G00214719 (2011).

15. Engel, Y., Etzion, O., Feldman, Z.: A basic model for proactive event-driven computing. In: Proceedings of the 6th ACM international conference on distributed event-based systems, pp. 107-118. ACM (2012).

16. Bousdekis, A., Mentzas, G.: A Multiple Criteria Approach Using ELECTRE for the Selection of Maintenance Strategy in Manufacturing Companies. In: 5th International Symposium - 27th National Conference on Operational Research, pp. 117-121, HELORS (2016). 